\title{
Luminescence of Excitons in Slightly Asymmetric Double Quantum Wells
}

\author{
(C) A.V. Akimov, E.S. Moskalenko, A.L. Zhmodikov, D.A. Mazurenko, A.A. Kaplyanskii, L.J. Challis*, \\ T.S. Cheng*, C.T. Foxon* \\ A.F.loffe Physical-Technical Institute, Russian Academy of Sciences, \\ 194021 St.-Petersburg, Russia \\ * Physics Department, Nottingham University, University Park NG7 2RD, Nottingham, UK
}

(Поступила в Редакцию 26 ноября 1996 г.)

We report the first studies of exciton luminescence spectra from asymmetric double quantum wells (DQWs) of very similar width. The DQWs were of GaAs/AlGaAs and the differences in widths of the coupled wells were one or two monolayers. The coupled direct and indirect exciton states anticross with a resonance splitting of $1.33 \mathrm{meV}$. An additional luminescence line appearing at low temperatures is identified as a localized indirect exciton.

An asymmetric double quantum well (DQW) in which the two QWs are separated by a narrow barrier gives rise to a minimum of four luminescence lines. Two of these are due to recombination of so-called direct exciton (DX) states in which the electron and hole are in the same quantum well and two to indirect exciton (IX) states in which the electron and hole are in different wells [1-6]. A number of optical studies have been made of these systems and of the changes in line positions when the relative positions of the energy levels in the two QWs are shifted by an applied electric field $F$. The energies of the indirect excitons vary approximately linearly with $F$ while those of the direct excitons are approximately fixed. As a result, the DX and IX lines cross or rather anticross since the electrons involved in both emission processes can tunnel through the barrier separating the wells. (In principle this can also happen for holes but in this case the tunnelling rate is too small to produce measurable effects.)

In previous studies of such systems [1-17], the widths of the coupled quantum wells differed by several monolayers leading to differences in the DX energies of $10 \mathrm{meV}$ or more. In the present work however, the DQWs are only slightly asymmetric, the widths of the coupled QWs differing by only 1 or 2 monolayers (MLs). The main purpose of working with such systems is that they have the potential to operate as tunable spectrometers of non-equilibrium acoustic phonons in the important range below $1000 \mathrm{GHz}$ in which phonons propagate ballistically through material such as GaAs allowing the possibility of detailed study of relaxation and other processes. This work will be discussed in a later paper. In the course of investigating these systems however we have found that the spectroscopic properties of the devices used are very well defined and, of particular note, provide the first observation of indirect exciton localization. It is that aspect of the work that is reported here.

\section{Samples and experimental details}

Samples were taken from two wafers, NU1116 and NU1117, each consisting of a $1 \mu \mathrm{m} \mathrm{GaAs}$ buffer layer followed by three DQWs. The structures were grown by MBE at $T=630^{\circ} \mathrm{C}$ on $0.4 \mathrm{~mm}$ thick (001) semi-insulating
GaAs substrates. Fig. 1 shows the growth scheme for wafer NU1117. The barriers are all of $\mathrm{Al}_{0.33} \mathrm{Ga}_{0.67} \mathrm{As}$ and the QWs of GaAs. The thickness $b$ of the narrow barriers between two QWs is $38.2 \AA$ and that of the thicker barriers between DQWs is $203.5 \AA$. The widths $a_{w}, a_{n}$ of the QWs differed by 1ML in NU1116 $\left(a_{w}-a_{n}=2.85 \AA\right)$ and 2MLs in NU1117 $\left(a_{w}-a_{n}=5.7 \AA\right)$ with layer widths $\left(a_{w} / b / a_{n}\right.$ in $\AA$ ) of $101.8 / 38.2 / 99.0,90.5 / 38.2 / 87.6$ and 82.0/38.2/79.1 in NU1116 and 200.7/38.2/195.0, 101.8/38.2/96.1 and 82.0/38.2/76.3 in NU1117. All the DQWs showed similar results and in the present paper we concentrate on those obtained with the 101.8/38.2/96.1 DQW.

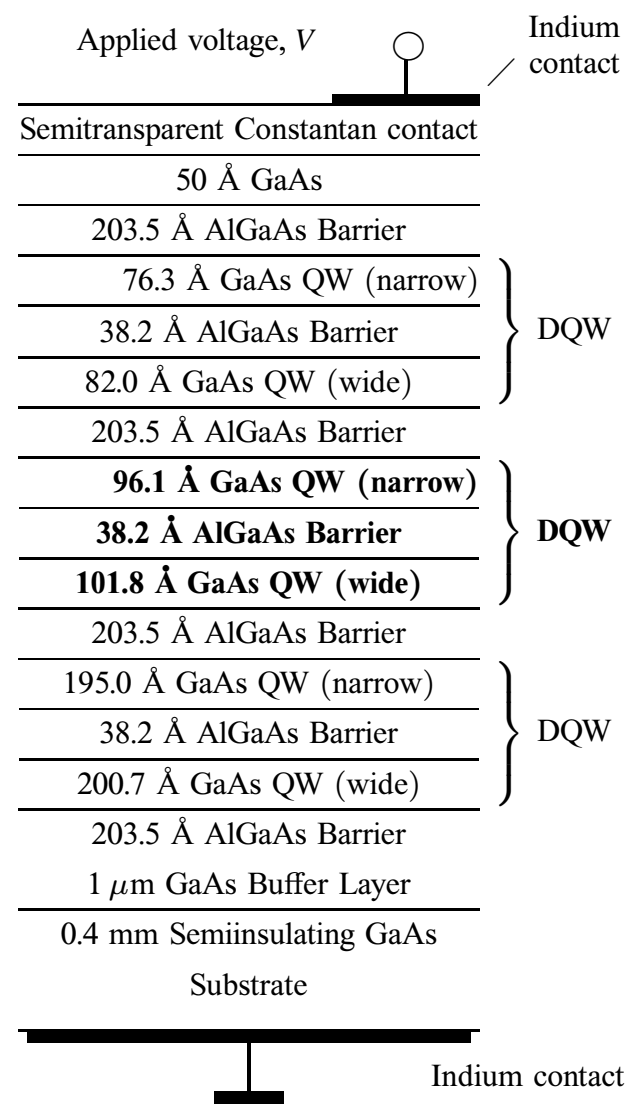

Figure 1. The layer structure of wafer NU1117. 


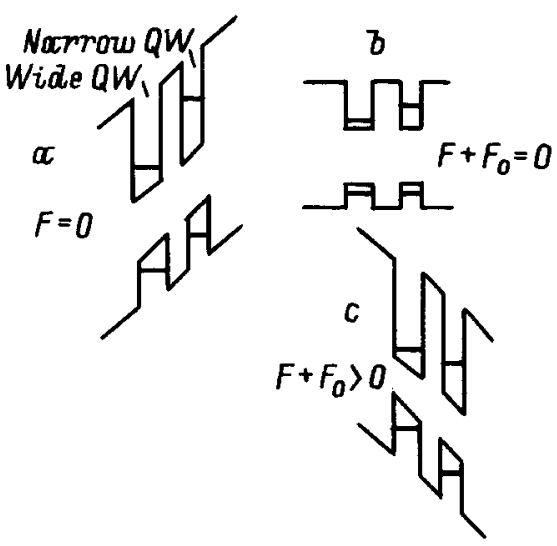

Figure 2. The band diagram of a DQW. $a-$ for zero applied field (internal field $F_{0}$ only), $b$ - under flat band conditions $\left(F+F_{0}=0\right), c-$ for positive net field $\left(F+F_{0}>0\right)$.

The experiments were carried out in a continuous flow helium cryostat with temperature control of $\pm 0.01 \mathrm{~K}$. The luminescence was excited by a $\mathrm{He}-\mathrm{Ne}$ laser beam $(\lambda=730 \mathrm{~nm})$ focussed down to $100 \mu \mathrm{m}$ diameter. The power density at the surface of the sample was $<1 \mathrm{~W} / \mathrm{cm}^{2}$ and underbarrier optical excitation was used to avoid luminescence from excitons bound to impurities in the barriers. The luminescence was analysed by a double monochromator with $0.2 \mathrm{meV}$ spectral resolution and detected by a photomultiplier followed by a photon counting system.

As already noted, the relative energy positions of the electron levels in a DQW are strongly affected by electric fields $[1-17]$ and we have used this to make a detailed examination of the luminescence as a function of energy level separation. DQWs in fact invariably contain an internal electric field $F_{0}$ (Fig. 2, $a$ ) whose magnitude and direction depend mainly on the doping of the GaAs/AlGaAs layers. In the arrangement normally used for electric field experiments an undoped DQW is located between $p$ - and $n$-layers and $F_{0}$ is determined by space charge effects. However the doping of the $p$ and $n$ layers leads to exciton luminescence lines too broad (up to $10 \mathrm{meV}$ ) for the resolution required here and so, in the present work, nominally undoped material was used with residual impurity concentration in the GaAs of $n \sim 10^{14} \mathrm{~cm}^{-3}$. A semitransparent constantan film was evaporated above the DQWs to form a Schottky contact (Fig. 1) and an indium film was deposited on the back side of the substrate. No change in luminescence spectrum could be detected after the contact deposition and the spectral width of the exciton lines at helium temperatures remained $\leqslant 1 \mathrm{meV}$.

The internal electric field in these structures changes with optical excitation by an amount which varies with both intensity and lattice temperature $T$ and it seems likely that the field is associated with space charge in the buffer layer/GaAs substrate interface and Fermi level pinning at the surface although this has not been established. The total field can be modified by applying a voltage $V$ between the contacts and, by adjusting $V$ to a particular value $V=V_{f}$, the internal electric field can be compensated and "flat band" conditions obtained: $F+F_{0}=0$ (Fig. $2, b$ ). The corresponding band diagram for $V>V_{f}$ for the DQW used in our experiments is shown schematically in Fig. 2,c.

To analyse the experimental results it is necessary to know how $F$ depends on the voltage $V$ applied to the contacts. Fortunately, as is shown in Sec. 2.A, there is a wide range of $V$ where a linear dependence $F(V)=k V$ is observed with $k=7 \cdot 10^{3} \mathrm{~cm}^{-1}$ for $T=10-20 \mathrm{~K}$ and $V>1 \mathrm{~V}$ although this is not the case over the whole experimental range. $k$ decreases with temperature below $10 \mathrm{~K}$ and, for example, at $T=5 \mathrm{~K}, k=3 \cdot 10^{3} \mathrm{~cm}^{-1}$. The sign of the internal field is such that a positive voltage of the Schottky contact is needed to compensate $F_{0}$ and from the size of $k$ we conclude that one DQW has a resistance comparable with $0.4 \mathrm{~mm}$ of semi-insulating GaAs, a somewhat surprising result.

\section{Experimental results and discussion}

A. Anticrossing of Direct and Indirect Exciton States. The luminescence spectrum of DQW 101.8/38.2/96.1 consists of several narrow $(\leqslant 1 \mathrm{meV})$ lines whose width and energy correspond to exciton recombination. The luminescence spectrum depends strongly with $V$ and $T$ in both line position and intensity and measurements at $T=20 \mathrm{~K}$ are shown in Fig. 3, $a$ for several values of $V$. Four excitonic lines are observed of which line 1 is dominant and, for $V<2 \mathrm{~V}$, has energy, $h \nu_{1}=1.5551 \mathrm{eV}$, in good agreement with that calculated for a direct heavyhole exciton in the wider $(101.8 \AA)$ QW. The line shifts to lower energy as $V$ increases (Fig. 3, $a$ ) and for $V>2 \mathrm{~V}$, a second line 2 , appears from the high energy side, increases in intensity and approaches an energy equal to the initial energy of line 1 . The energy, $1.5589 \mathrm{eV}$, of line 4 corresponds to that of a direct heavy-hole exciton in the narrower $(96.1 \AA)$ QW at low voltages but shifts to higher energies and weaker intensities as $V$ is increased and another line, 3, appears whose energy approaches the initial energy of line 4 .

Only two lines, 1 and 3 , are detectable at lower temperatures and this is illustrated by the data in Fig. 3, $b$ for $T=5 \mathrm{~K}$. However for $V>3 \mathrm{~V}$ another line, $L$, appears $\sim 1 \mathrm{meV}$ lower in energy than line 1 which it "accompanies" as it shifts to lower energies with increasing $V$. Line 1 disappears for $V>3.7 \mathrm{~V}$ but line $L$ continues to follow line $I$ 's high temperature position always being $\sim 1 \mathrm{meV}$ lower in energy. Its possible origin is discussed later.

The origin and behaviour of lines 1-4 seem readily explained in terms of DX and IX recombination as shown in Fig. 4, $a$. The DX lines are referred to as DXW (wide QW) and DXN (narrow QW) and their excitonic energies $(\Gamma$-point $)$ are given respectively by

$$
E_{x w}^{D}=E_{e w}-E_{h w}-E_{B}^{D}, \quad E_{x n}^{D}=E_{e n}-E_{h n}-E_{B}^{D},
$$

where $E_{e w}, E_{e n}, E_{h w}, E_{h n}$ are the electron and hole energies in the wide and narrow QWs respectively and $E_{B}^{D}$ is the binding 


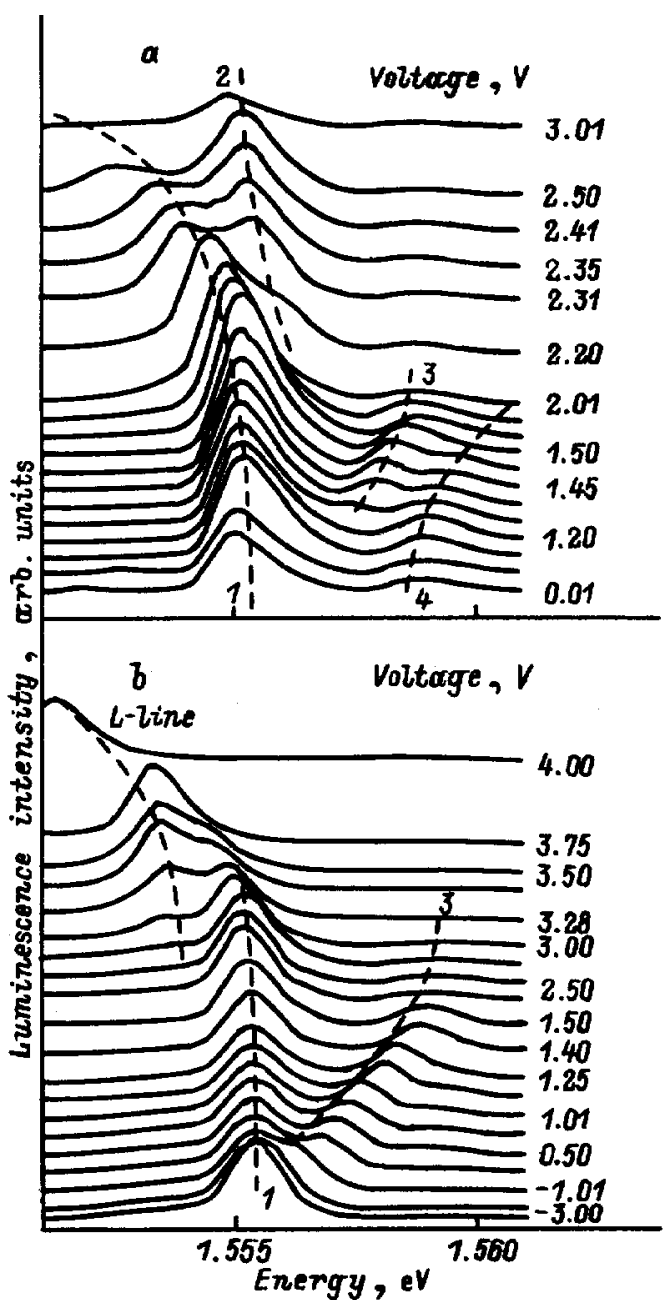

Figure 3. Luminescence spectrum of DQW measured at $20(a)$ and $5 \mathrm{~K}(b)$ for different applied voltages $V$. The dotted lines are drawn for the eye to follow the peak position of the exciton luminescence lines.

energy of DX. The quadratic Stark effect is negligible for $F+F_{0}<10 \mathrm{kV} / \mathrm{cm}$ so that, as noted, the energies $E_{x w}^{D}$, $E_{x n}^{D}$ are essentially independent of $V$ and only depend on the widths, $a_{n}$ and $a_{w}$.

The energies of the indirect excitons equal

$$
E_{x w}^{I}=E_{e n}-E_{h w}-E_{B}^{I}, \quad E_{x n}^{I}=E_{e w}-E_{h n}-E_{B}^{I},
$$

where $E_{B}^{I}$ equals their binding energy and the subscript in each expression, $(x w)$ or $(x n)$, indicates the location of the hole ( $w=$ wide QW, $n=$ narrow QW). Previous measurements $[3,4,16,17]$ have shown that a pure IX state has a linear Stark effect so that

$$
\begin{aligned}
& E_{x w}^{I}=E_{e n}^{0}-E_{h w}^{0}-e\left(F+F_{0}\right) z-E_{B}^{I}, \\
& E_{x n}^{I}=E_{e w}^{0}-E_{h n}^{0}+e\left(F+F_{0}\right) z-E_{B}^{I},
\end{aligned}
$$

where $E_{e n(w)}^{0}$ and $E_{h n(w)}^{0}$ are the energies of the electrons and holes under flat band conditions $\left(F+F_{0}=0\right)$. From Eqs.(1) and (3) it is seen that the DXW(N) and $I X W(N)$ levels should cross, $E_{x w(n)}^{D}=E_{x w(n)}^{I}$, (Fig. 4,b) when the applied field $F_{r w(n)}$ equals

$$
\begin{aligned}
& F_{r w}=\frac{1}{e z}\left(\Delta E_{e}^{0}+E_{B}^{D}-E_{B}^{I}\right)-F_{0}, \\
& F_{r n}=\frac{1}{e z}\left(\Delta E_{e}^{0}-E_{B}^{D}+E_{B}^{I}\right)-F_{0},
\end{aligned}
$$

where $\Delta E_{e}^{0}=E_{e n}^{0}-E_{e w}^{0}$. However the coherent electron tunnelling that can take place at or near resonance leads to anticrossing and so to splitting into symmetric and antisymmetric combinations of the DXN and $I \mathrm{XN}$ and of DXW and $I$ XW eigenstates as shown in Fig. 4, $c$ for DXN and IXN states. The energy splitting $\delta \sim h / \tau_{e}$, where $\tau_{e}$ is the electron tunnelling time, and in these combined states the electron is shared between the two QWs while remaining bound to a localized hole in one of the QWs. $F_{r w(n)}$ differs somewhat from the field $F_{r e}$ at which the electron levels in the wide and narrow QWs are in resonance $\left(F_{r e}+F_{0}=\frac{1}{e z} \Delta E_{e}^{0}\right)$ because of excitonic effects; this difference appears to have been overlooked in some of the earlier papers $[3,4,6,16,17]$. When anticrossing is included, the expressions for the energy levels [6] become

$$
\begin{aligned}
& E_{1,2}=\frac{1}{2}\left\{E_{x w}^{D}+E_{x w}^{I} \pm\left[\left(E_{x w}^{D}-E_{x w}^{I}\right)^{2}+\delta^{2}\right]^{1 / 2}\right\}, \\
& E_{3,4}=\frac{1}{2}\left\{E_{x n}^{D}+E_{x n}^{I} \pm\left[\left(E_{x n}^{D}-E_{x n}^{I}\right)^{2}+\delta^{2}\right]^{1 / 2}\right\}
\end{aligned}
$$

and we note that the anticrossing has previously been observed in photoconductivity [16], optical absorption [6] and photoluminescence [18] experiments.

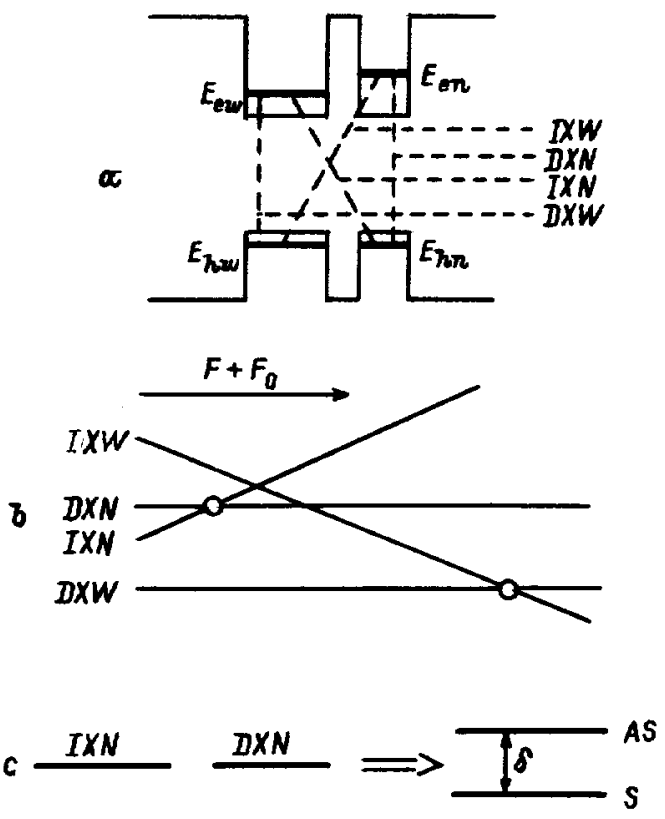

Figure 4. a) The band diagram and exciton transitions of the DQW. $b$ ) The dependence (schematic) of the exciton energies on applied field $F$; the circles show the DX/IX anticrossing regions. c) A diagram showing the level splitting between the symmetric $\langle S|$ and antisymmetric $\langle A S|$ eigenstates at the anticrossing. 


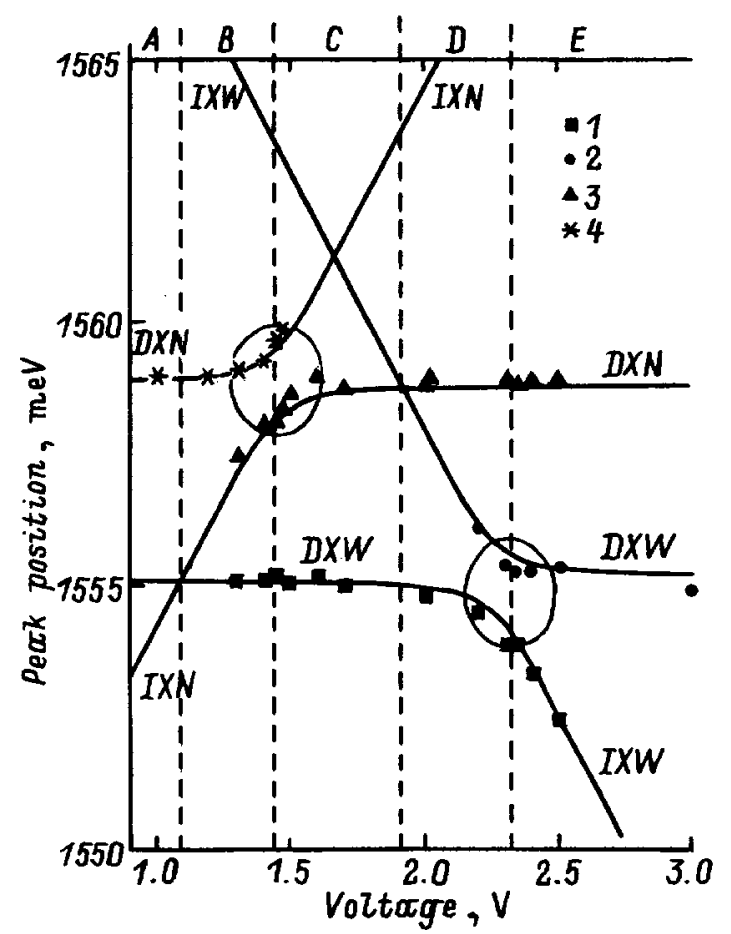

Figure 5. The measured (symbols) and calculated (solid curves) energies of the exciton lines as a function of applied voltage at $20 \mathrm{~K}$. The circles show the DX/IX anticrossing regions.

Lines $1-4$ of the luminescence spectrum shown in Fig. 3 seem consistent with these predictions and this can be seen more clearly in Fig. 5 which shows their energies at $T=20 \mathrm{~K}$. The symbols show the experimental data and the solid curves are calculated [Eq.(5)] using $E_{x w}^{D}=1.5551 \mathrm{eV}$, $E_{x n}^{D}=1.5589 \mathrm{eV}$, and $\delta=1.33 \mathrm{meV}$. The two anticrossing regions shown circled occur at "resonant" voltages $V_{r n}=1.4 \mathrm{~V}$ and $V_{r w}=2.3 \mathrm{~V}$. As noted earlier, for $V>1 \mathrm{~V}$, the data are consistent with a linear field dependence $F(V)=\mathrm{k} V$ with $k=7 \cdot 10^{3} \mathrm{~cm}^{-1}$ at $20 \mathrm{~K}$ and a similar value is found at $10 \mathrm{~K}$ although it decreases at lower temperatures and for $T=5 \mathrm{~K}, k=3 \cdot 10^{3} \mathrm{~cm}^{-1}$. From the data and calculated values for $E_{B}^{D}$ in DQWs of $8 \mathrm{meV}$ [16] and for $\Delta E_{e}^{0}$ of $3.32 \mathrm{meV}$ we obtain a value for the binding energy of $I \mathrm{X}$ of $E_{B}^{I}=3.3 \mathrm{meV}$ in good agreement with previous values for similar samples $[3,4,16,17]$. The voltage $V_{f}$ at which $F+F_{0}=0$ at $20 \mathrm{~K}$ is $1.54 \mathrm{~V}$.

The intensities of lines $1-4$ are governed by the tunnelling and by the relaxation processes of DX and IX through the emission and absorption of acoustic phonons. The results show that the exciton states DXN and IXN are in thermal equilibrium as are states DXW and IXW. However the thermal equilibrium between these two pairs is not established [19]. Detailed analysis of the luminescence intensities and relaxation processes will be published shortly.

B. The origin of line $L$ and changes in the nonradiative decay of excitons. The experimental spectrum at $5 \mathrm{~K}$ in Fig. $3, b$ shows the appearance of a luminescence line $L$ when $V>3 \mathrm{~V}$ which has not previously been reported. As $V$ increases, line $L$ remains red-shifted from $1, I \mathrm{XW}$, by an approximately constant energy, $1 \mathrm{meV}$, but grows in size while line 1 falls. We attribute line $L$ to a localized indirect exciton (LIX): the indirect exciton 1 bound to an impurity or interface defect. Support for this comes from the size of the redshift which is close to the binding energy of bound excitons in GaAs QWs [20]. Line $L$ disappears for $T>10 \mathrm{~K}$ while line 1 remains and this is consistent with the dissociation of localized excitons (which have only a small density of states) into free excitons with increasing temperature.

The reason why line $L$ only appears for $V>3 \mathrm{~V}$ and increases in intensity at the expense of line 1 as $V$ increases seems explicable in terms of the lifetime of the IXW state. At high voltages this is a pure IX state and since this has the lowest exciton energy it has a long lifetime [1] and so a good chance of being captured by a defect before annihilation. However, as $V$ decreases, IXW moves towards the anticrossing so there is an increasing admixture of DXW into the IXW state. This leads to a steady decrease in the radiative lifetime and hence in the chance of capture before annihilation. It seems likely that the IXN exciton should also become localized at low voltages where it has the lowes energy and this should lead to a line approximately $1 \mathrm{meV}$ below 3. No evidence of this can be seen in Fig. 3 but this may be because of difficulties in resolving it from line 3 in the region where lines 1 and 3 cross.

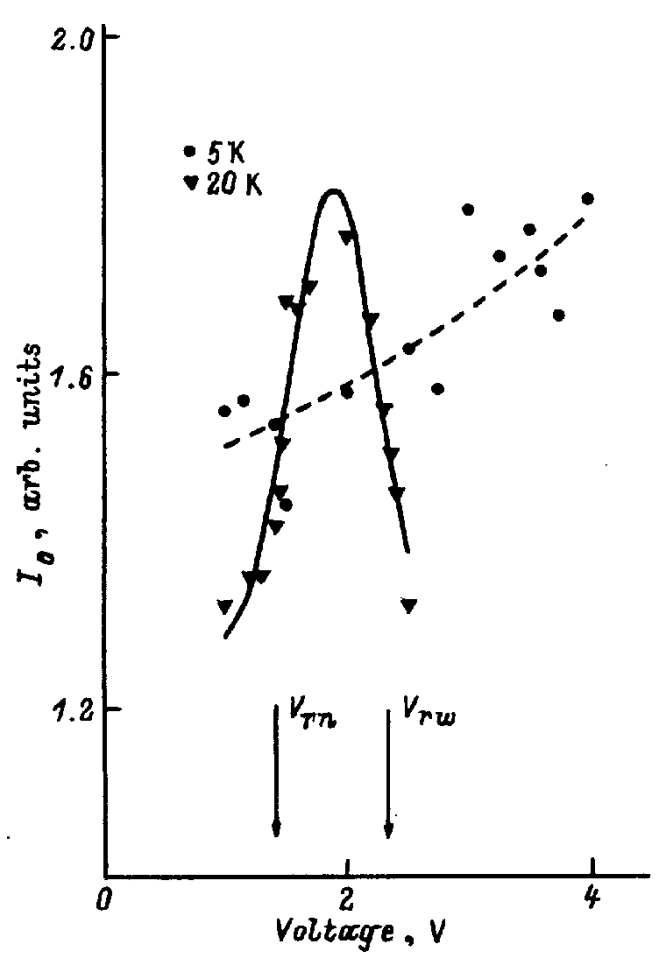

Figure 6. The dependence of the total intensity $I_{0}$ on applied voltage for $T=5$ and $20 \mathrm{~K}$. Since $F_{0}$ and $k$ depend on temperature, the relative values of $I_{0}$ at the two temperatures at $2 \mathrm{~V}$ are not comparable. The arrows show the positions $V_{r n}$ and $V_{r w}$ of the DX/IX resonances for $T=20 \mathrm{~K}$. The lines through the two sets of data are to guide the eye. 
Further evidence of indirect exciton localization can be seen in the voltage variation of the total intensity $I_{0}$ obtained by integration over the whole spectral range. Fig. 6 shows that this variation is strongly temperature dependent and we first discuss the data at $20 \mathrm{~K}$ which is too high for any significant localization. There are pronounced decreases in $I_{0}$ for $V<V_{r n}$ and $V>V_{r w}$ where the lowest energy excitons are $I \mathrm{XN}$ and $I \mathrm{XW}$ (Fig. 5). These have much longer radiative lifetimes than DX excitons [1] so that a much higher proportion of them decay nonradiatively accounting for the decreases in $I_{0}$ observed. Strikingly different behaviour however is observed at $5 \mathrm{~K}$. There are now no decreases in $I_{0}$ for $V<V_{r n}$ and $V>V_{r w}$ suggesting that the lowest energy states are no longer the free indirect exciton states IXN and IXW but states which largely decay radiatively. This is entirely consistent with indirect exciton localization. It is well-known that the probability of nonradiative decay from direct excitons is much reduced by localization, the greater confinement of the exciton wavefunction reducing the overlap with nonradiative decay centres, and it seems very probable that a similar effect should occur for indirect excitons. We conclude therefore that, at $5 \mathrm{~K}$, the majority of $I \mathrm{XN}$ and $I \mathrm{XW}$ excitons become localized when $V<V_{r n}$ and $V>V_{r w}$.

The luminescence spectrum of slightly asymmetric DQWs has been shown to contain four free exciton lines: two direct and two indirect. The indirect excitons become localized (LIX) at low temperatures, $T<10 \mathrm{~K}$. One of the LIX gives rise to a new line, $L$, while the presence of the other is inferred from the decrease in proportion of non-radiative decay that occurs on localization. Anticrossing has been observed between the direct and indirect excitons and, at resonance, the symmetric and antisymmetric eigenstates are separated by $1.3 \mathrm{meV}$.

We gratefully acknowledge financial support from the Russian Foundation for Fundamental Studies (96-0216952a) and the European Commission (INTAS-94-395).

\section{References}

[1] T.B. Norris, N. Vodjdani, B. Vinter, C. Weisbush, G.A. Mourou. Phys. Rev. B40, 2, 1392 (1989).

[2] M. Nido, M.G.W. Alexander, W.W. Rühle, K. Köhler. Phys. Rev. B43, 2, 1839 (1991).

[3] R. Ferreira, C. Delalande, H.W. Liu, G. Bastard, B. Etienne, J.F. Palmier. Phys. Rev. B42, 14, 9170 (1990).

[4] R. Ferreira, P. Rolland, Ph. Roussignol. C. Delalande, A. Vinattieri, L. Carraresi, M. Colocci, N. Roy, B. Sermage, J.F. Palmer, B. Etienne. Phys. Rev. B45, 20, 11782 (1992).

[5] J.E. Golub, K. Kash, J.P. Harbison, L.T. Florez. Phys. Rev. B41, 12, 8564 (1990); M.R. Reshotko, L.D. Shvartsman, J.E. Golub. Phys. Rev. B50, 3, 4692 (1994).

[6] K. Leo, J. Shah, E.O. Gobel, T.C. damen, S. Schmitt-Rink, W. Scäfer, K. Köhler. Phys. Rev. Lett. 66. 2, 201 (1991).

[7] D.Y. Oberli, J. Shah, T.C. Damen, T.Y. Chang, C.W. Tu, D.A.B. Miller, J.E. Henry, R.F. Kopf, N. Sauer, A.E. DiGiovanni. Phys. Rev. B40, 5, 3028 (1989).
[8] H.W. Liu, R. Ferreira, G. Bastard, C. Delalande, J.F. Palmier, B. Etienne. Appl. Phys. Lett. 54, 21, 2082 (1989).

[9] F. Clérot, B. Deveaud, A. Chomette, A. Regreny, B. Sermage. Phys. Rev. B41, 9, 5756 (1990); B. Deveaud, A. Chomette, F. Clérot, P. Auvray, A. Regreny, R. Ferreira, G. Bastard. Phys. Rev. B42, 11, 7021 (1990).

[10] T.B. Norris, N. Vodjdani, B. Vinter, E. Costard, E. Böckenhoff. Phys. Rev. B43, 2, 1867 (1991).

[11] R. Strobel, R. Eccleston, J. Kuhl, K. Köhler. Phys. Rev. B43, 15, 12564 (1991).

[12] Ph. Roussignol, A. Vinattieri, L. Carraresi, M. Colocci, A. Fasolino. Phys. Rev. B44, 16, 8873 (1991).

[13] A.P. Heberle, W.W. Rühle, M.G.W. Alexander, K. Köhler. Semicond. Sci. Technol. 7, B421 (1992).

[14] A.P. Heberle, X.Q. Zhou, A. Tackeuchi, W.W. Rühle, K. Köhler. Semicond. Sci. Technol. 9, 519 (1994).

[15] R.G. Ispasoiu, A.M. Fox, C.T. Foxon, J.E. Cunningham, W.Y. Jan. Semicond. Sci. Technol. 9, 545 (1994).

[16] A.M. Fox, D.A.B. Miller, G. Livescu, J.E. Cunningham, W.Y. Jan. Phys. Rev. B44, 12, 6231 (1991).

[17] H. Schneider, J. Wagner, K. Ploog. Phys. Rev. B48, 15, 11051 (1993).

[18] O. Brandt, K. Kanamoto, Y. Tokuda, Y. Abe, Y. Wada, N. Tsukada. J. Appl. Phys. 75, 4, 2105 (1994).

[19] A.V. Akimov, E.S. Moskalenko, A.L. Zhmodikov, D.A. Mazurenko, A.A. Kaplyanskii, L.J. Challis, T.S. Cheng, C.T. Foxon. Nanostructures: Physics and Technology'96. Int. Symp. Abstracts of Invited Lectures and Contributed Papers. St.Petersburg, Russia (24-28 June 1996). P. 86.

[20] D.C. Reynolds, K.R. Evans, K.G. Merkel, C.E. Stutz, P.W. Yu. Phys. Rev. B43, 11, 9087 (1991). 